\title{
Une source de rayonnement développée pour des mesures optiques de spectroscopie d'absorption large bande
}

\author{
D. Hong, G. Sandolache, T. Capelle, J.M. Bauchire, E. Le Menn et C. Fleurier \\ GREMI, Université d'Orléans, 14 rue d'lssoudun, BP. 6744, 45067 Orléans cedex 2, France
}

\begin{abstract}
Résumé. Afin d'étudier la période post-arc et les gaz entourant l'arc électrique dans les disjoncteurs, une source de rayonnement large bande a été développée pour effectuer des mesures de spectroscopie d'absorption. La source a été étudiée afin de déterminer les meilleures conditions produisant un spectre intense et continu de rayonnement dans le domaine ultra-violet et également dans le visible. Des essais d'absorption ont été réalisés dans un disjoncteur basse tension de type rail. L'absorption des raies de résonance de cuivre (324.7 et $327.4 \mathrm{~nm}$ ) a permis de déterminer la densité d'atomes de cuivre dans la région à l'arrière de l'arc. En outre, l'absorption des bandes de $S$ wan de $\mathrm{C}_{2}$ a permis de déterminer la concentration de carbone et également la température cinétique du gaz chaud.
\end{abstract}

\section{INTRODUCTION}

La spectroscopie d'absorption optique (OAS) est une technique généralement utilisée pour diagnostiquer les milieux où l'émission lumineuse est nulle ou très faible. L'utilisation de la technique d'OAS pour des milieux transitoires rapides monocoup ou à faible taux de répétition et irrégulier, requiert des sources intenses ayant une émission spectrale de large bande. La période post-arc ou les gaz chauds entourant l'arc mobile dans les disjoncteurs appartiennent à cette classe de milieux transitoires rapides. Afin d'effectuer de telles mesures d'absorption, une source de rayonnement de large bande spectrale continue et d'intensité élevée a été développée. Elle consiste en une décharge électrique utilisant l'effet bien connu de Z-Pinch.

Avec une montée rapide $\left(\mathrm{dI} / \mathrm{dt}=10^{10} \mathrm{~A} / \mathrm{s}\right)$, le courant de décharge dans un tube cylindrique commence à circuler dans une couche mince sur la surface intérieure de la paroi du tube. Le plasma ainsi créé a donc la forme d'un cylindre creux. Ce cylindre de plasma est soumis à une force de Laplace résultant de l'interaction du courant avec le champ magnétique azimutal créé par le courant lui-même, et est par conséquent fortement accélérée vers l'axe de tube. Quand le plasma atteint l'axe, il devient très dense, résultat de l'effet chasse-neige qui balaye toutes les particules vers l'axe pendant la compression. En outre la température est fortement augmentée en raison de la conversion de l'énergie cinétique de la couche de courant en énergie thermique au moment du collapse. Ce moment est désigné sous le nom du temps de pinch.

Dans nos conditions spécifiques, une densité d'électrons $\mathrm{N}_{\mathrm{e}}$ supérieure à $10^{20} \mathrm{~cm}^{-3}$ est obtenue. En conséquence, d'une part, le Bremstrahlung et la recombinaison radiative, toutes les deux fonction de $\mathrm{Ne}_{\mathrm{e}}{ }^{2}$ produisent une émission continue très intense et, $d$ 'autre part, les raies spectrales sont fortement élargies par l'effet Stark. En addition de ces deux effets propices à la formation d'un rayonnement continu intense, l'observation de la lumière le long de l'axe du tube permet d'obtenir une grande épaisseur optique pour les raies spectrales comme pour le rayonnement continu, de sorte que le spectre émis est très voisin du rayonnement d'un corps noir à une température estimée à $80000 \mathrm{~K}$ d'après une simulation numérique.

Ce bref et intense rayonnement continu produit à l'instant du pinch est très approprié aux mesures d'OAS dans les disjoncteurs. Des essais de mesures d'absorption ont été réalisés sur un disjoncteur basse tension de type rail [1], où un arc électrique se déplace le long de deux électrodes parallèles vers une zone d'extinction. Dans certaines cas, un arc de re-claquage peut se produire avant la zone d'extinction et causer l'échec de l'opération de coupure du courant. Des investigations sur les phénomènes de reclaquage ont été réalisées par C. Fiévet et al [1]. Il s'avère que le gaz chaud laissé par l'arc après son passage joue un rôle majeur dans le processus de re-claquage. La technique d'OAS est bien adaptée à la mesure de composition, de température de ce gaz chaud et des concentrations de chaque espèce. 


\section{Z-pinch}

Le montage expérimental du Z-pinch utilisé dans ce travail ainsi que le montage de mesure optique sont schématisés sur la figure 1. Le plasma est créé dans un tube cylindrique en quartz de diamètre intérieur de $10,8 \mathrm{~cm}$. La distance entre les deux électrodes de carbone est de 20 centimètres. Un trou de 1 centimètre de diamètre est percé au centre de chaque électrode pour permettre des mesures optiques le long de l'axe du tube et pour faciliter l'écoulement continu de gaz (argon) dont le débit est contrôlé par un régulateur de débit massique. Le courant de décharge est transporté par 12 câbles coaxiaux distribués cylindriquement sur le tube de décharge.

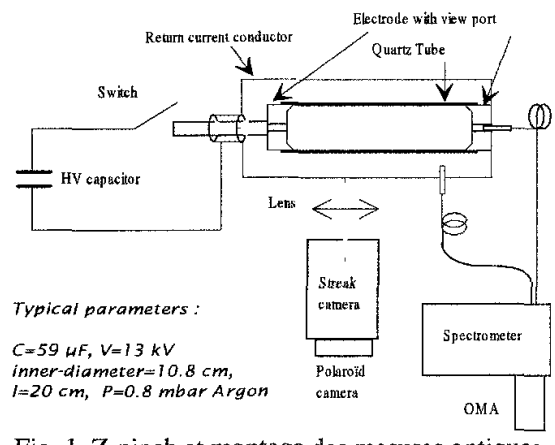

Fig. 1. Z-pinch et montage des mesures optiques,

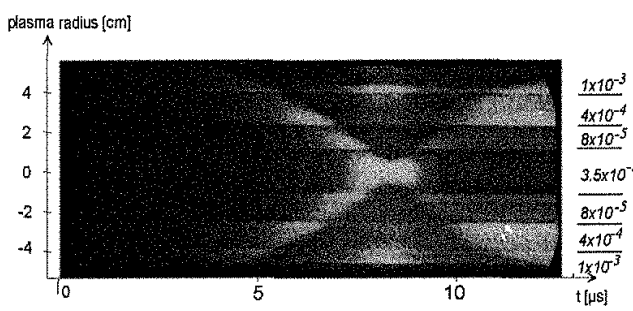

Fig. 2. Évolution temporelle du diamètre du plasma. La transmission de chaque bande d'atténuateur neutre est donnée à droite de la bande.

Une fibre optique couplée à une photodiode est utilisée pour mesurer l'évolution temporelle de la lumière du plasma. Une caméra à balayage de fente permet d'observer l'évolution temporelle et l'intensité d'une section transversale du plasma en fonction du temps et enfin un spectromètre équipé d'une caméra intensifiée est utilisé pour les enregistrements de spectre. La lumière du plasma est transmise par une fibre optique équipée d'une lentille pour collecter la lumière dans un angle solide correspondant au diamètre du plasma au moment du pinch (environ $1 \mathrm{~cm}$ ). Le temps d'ouverture de caméra est fixé à $2 \mu \mathrm{s}$, ce qui correspond à la durée d'impulsion de la lumière émise par le pinch. .

La figure 2 montre une photo obtenue par la caméra à balayage. L'axe horizontal correspond au temps tandis que l'axe vertical correspond au diamètre de plasma. En raison de la forte variation de la brillance de la source durant la décharge, une atténuation différentielle a dû être utilisée pour ces mesures. Elle consiste en un empilement de bandes d'atténuateurs neutres placés sur la fente d'entrée de la caméra afin d'obtenir une atténuation très importante au centre de la photo qui correspond à l'axe du pinch. L'atténuation diminue par pas lorsqu'on s'éloigne du centre. En conséquence, la photo apparait comme une juxtaposition de bandes contiguës, chacune correspondant à un facteur de transmission spécifique indiqué par le nombre à la droite de la photo. Les conditions de travail sont toujours $13 \mathrm{kV}(5 \mathrm{~kJ}$ emmagasinée dans un banc de condensateur de $59 \mu \mathrm{F}$ ) et 0,8 mbar. Les photographies montrent clairement la phase de compression du plasma. Au début, la couche de courant (ou le piston magnétique) se décolle de la paroi puis il accélère pour atteindre une vitesse de $1,5 \mathrm{~cm} / \mu \mathrm{s}$ juste avant la compression maximale. Au temps de pinch $(8,4 \mu \mathrm{s})$, le diamètre de plasma est d'environ $1,2 \mathrm{~cm}$, correspondant à un taux de compression de 9 , et le signal de photodiode mesurant le rayonnement du plasma montre un pic géant de lumière à cet instant.

Les spectres résolus en temps dans un intervalle spectral compris entre 328 et $340 \mathrm{~nm}$ ont été mesurés à plusieurs instants pendant la décharge et sont montrés dans la figure 3 . Le retard de déclenchement de la caméra est indiqué à la droite de chaque spectre. Entre 2 et $4 \mu \mathrm{s}$, l'intensité spectrale est négligeable. A $6 \mu \mathrm{s}$, le plasma arrive sur l'axe et l'émission dans la gamme 328 à $340 \mathrm{~nm}$ est dominée par les raies d'Ar III $(328,61,331,16,333,61,334,47,335,84 \mathrm{~nm}$, etc $)$. Le temps de pinch se produit $2 \mu \mathrm{s}$ plus tard. A ce moment là, l'émission de continuum devient très intense - l'échelle verticale du spectre $\mathrm{d}$ ) est 10 fois plus grande que celle des autres spectres- tandis que les raies spectrales disparaissent totalement en raison de la densité électronique très élevée du plasma. Le rayonnement du plasma est très proche de celui d'une émission de corps noir. Entre 14 et $16 \mu \mathrm{s}$, les raies d'Ar $\Pi(335,09,337,7$, $338,85 \mathrm{~nm}$, etc.) apparaissent tandis que les raies d'Ar III sont encore présentes. 
Le spectre dans la gamme 350 à $750 \mathrm{~nm}$, mesuré au moment du pinch, est présenté sur la figure 4 . Les fluctuations d'intensité sont en grande partie inhérentes à notre détecteur.

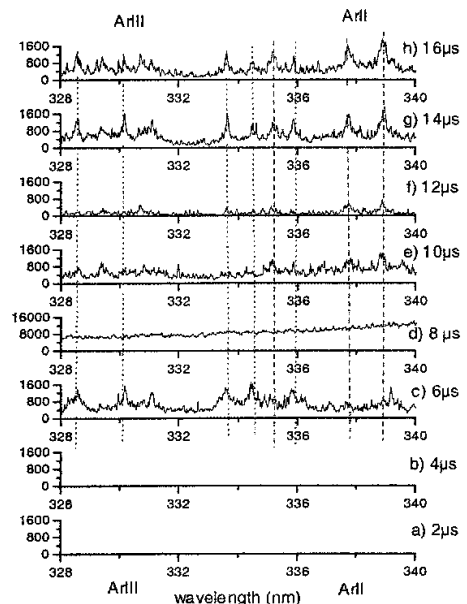

Fig. 3. Spectres résolus en temps (ouverture $=2 \mu \mathrm{s}$ ). Le retard est mentionné à droite de chaque spectre. L'échelle verticale du spectre d) est 10 fois plus grande que celle des autres spectres.

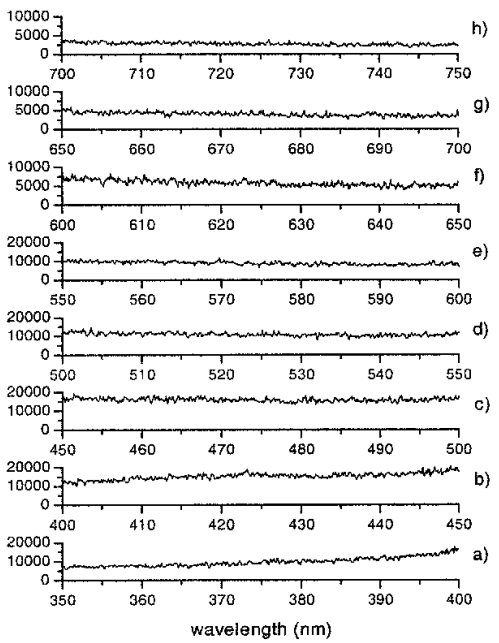

Fig. 4. Spectre dans la gamme de $350-750 \mathrm{~nm}$ au temps de pinch.

\section{MESURE D'ABSORPTION}

Les mesures d'absorption ont été effectuées avec le montage représenté en figure 5. Ce montage a été conçu pour étudier la coupure dans des disjoncteurs basse tension par des méthodes électriques et optiques.

Au démarrage le courant initial circule dans le circuit CSM avec une forme sinusoïdale amortie ayant une pseudo-période de $20 \mathrm{~ms}(50 \mathrm{~Hz})$. Le contact mobile $M$ est ouvert $1 \mathrm{~ms}$ après le démarrage du courant pour simuler la coupure, et un arc est alors créé entre $\mathrm{M}$ et $\mathrm{K}$. Sous l'effet de la force de Laplace, l'arc est alors transféré de $\mathrm{M}$ à $\mathrm{A}$ et se déplace ensuite entre les deux rails dans la direction de la chambre d'extinction. Cette opération provoque une forte érosion des électrodes de cuivre et des parois. Ainsi le gaz chaud à l'arrière de l'arc se compose d'air, de cuivre, et d'autres espèces, généralement du carbone et de l'hydrogène car les parois sont généralement constituées de matière plastique. Ces gaz chauds jouent un rôle important dans le phénomène de re-claquage et il est donc très utile d'en déterminer la composition et
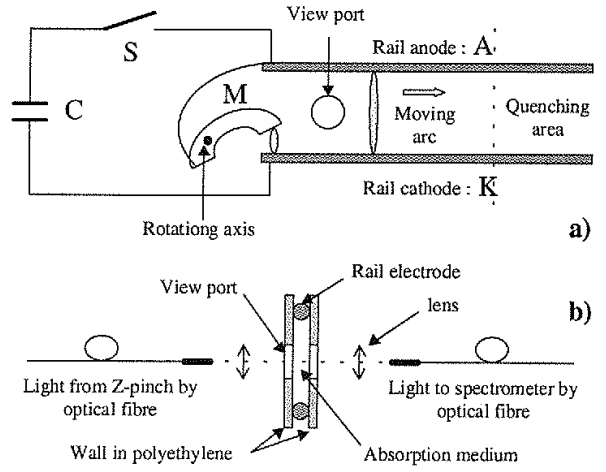

Fig. 5. Disjoncteur basse tension à rails et montage de mesure optique pour la spectroscopie d'absorption. la température pour les modèles simulant le fonctionnement du disjoncteur.

Dans la figure 6-a), un spectre d'absorption des deux raies de résonance du cuivre, $\mathrm{Cu}$ I 324,75 et $327,40 \mathrm{~nm}$, est présenté. La référence $\mathrm{I}_{\text {ref }}$ est l'intensité incidente non-absorbée et $\mathrm{I}_{\text {trans }}$ est lintensité transmise. Cette mesure a été effectuée à une distance de $6 \mathrm{~cm}$ du secteur d'allumage de l'arc et environ $0,5 \mathrm{~ms}$ après le passage de l'arc. Le rapport $R_{M}=I_{\text {trans }} / I_{\text {ref }}$ est tracé dans la figure 6 -b). La valeur théorique du coefficient d'absorption est indiquée par la relation $\alpha_{T}(\lambda)=\frac{\lambda^{4} A_{u l}}{8 \pi c} \frac{g_{u}}{g_{l}} N_{l} S(\lambda)$, où $S(\lambda)$ représente le profil 
normalisé théorique non absorbé de la raie et les symboles utilisés ont leurs significations habituelles ( $\lambda$ : longueur d'onde, $\mathrm{N}_{\mathrm{l}}$ : population de l'atome dans le niveau bas, etc.)

Afin de déterminer la concentration de cuivre en atome, le spectre expérimental $R_{M}$ a été comparé à un spectre synthétisé convolué avec la fonction d'appareil, en supposant que le profil théorique de la raie $S(\lambda)$ a une forme gaussienne: $S(\lambda)=\frac{1}{w} \sqrt{\frac{\ln 2}{\pi}} e^{\frac{\left(\lambda-\hat{-}_{0}\right)^{2}}{w^{2}} \ln 2}$. Le meilleur ajustement montré sur la figure 6-b) rapporte une densité d'atome de cuivre d'environ de $8,5 \times 10^{13} \mathrm{~cm}^{-3}$. On estime que la précision des mesures est de $20 \%$, principalement due aux fluctuations aléatoires du système de détection. Cette concentration élevée en atome de cuivre est de nature à favoriser le re-claquage de l'arc car leur potentiel d'ionisation est faible.

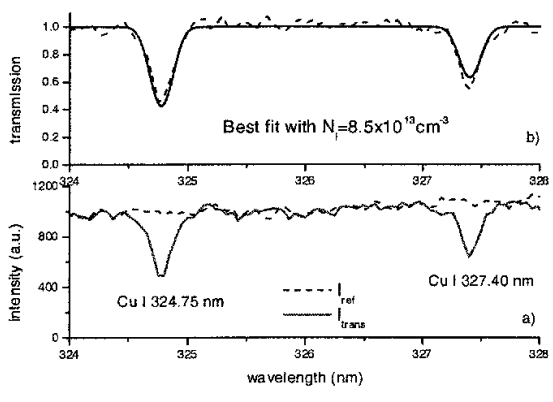

Fig. 6. a) Spectre d'absorption des raies de résonance de cuivre $\mathrm{Cu} I 324,75$ et $\mathrm{Cu} I 327,40 \mathrm{~nm}$; b) transmission et sa meilleure approche théorique.

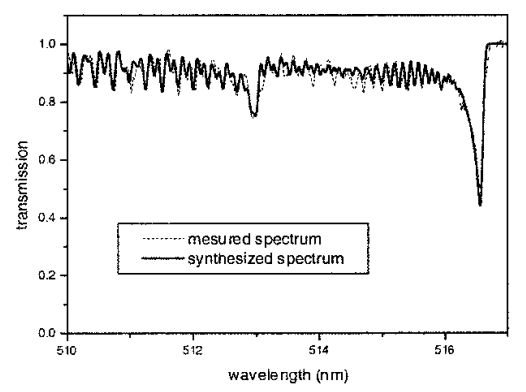

Fig. 7. Spectre d'absorption de $\mathrm{C}_{2}$ : bandes de $\operatorname{Swan}(0,0)$ et $\operatorname{Swan}(1,1)$.

Le spectre d'absorption des bandes de Swan de $\mathrm{C}_{2}(0,0)$ et $(1,1)$ est donné figure 7 . Les molécules de $\mathrm{C}_{2}$ proviennent de l'ablation des parois en plastique par l'arc. La simulation théorique de la structure de rotation et vibratoire de la bande moléculaire donne une concentration d'environ de $6,5 \times 10^{14} \mathrm{~cm}^{-3}$ et une température de rotation d'environ $3200 \mathrm{~K}$. La température cinétique du gaz, très voisine de la température de rotation, mesurée $0.5 \mathrm{~ms}$ après que le passage d'arc prouve que la vapeur est toujours très chaude ce qui peut également favoriser le re-claquage de l'arc.

\section{CONCLUSION}

La source lumineuse Z-pinch a été développée pour effectuer la spectroscopie d'absorption optique. La dynamique du pinch a été caractérisée au moyen d'une caméra à balayage de fente tandis que les spectres d'émission ont été enregistrés et analysés. Des conditions de travail optimales ont été définies pour obtenir un spectre intense et continu de rayonnement au temps de pinch.

Des essais d'utilisation de spectroscopie d'absorption optique, employant cette source, ont été réalisés sur le gaz et la vapeur chauds dans un disjoncteur basse tension à rails. L'absorption des raies de résonance du cuivre et des bandes moléculaires de Swan de $\mathrm{C}_{2}$ a permis d'estimer des densités de cuivre ou de carbone dans la région à l'arrière de l'arc électrique et démontre les capacités de notre source pour réaliser l'OAS. Ces mesures plutôt simples fournissent déjà des informations tout à fait intéressantes sur le gaz chaud derrière le disjoncteur. Des mesures plus précises et systématiques seront maintenant entreprises pour caractériser entièrement le disjoncteur pendant l'opération de coupure de courant.

Les auteurs remercient Schneider Electric pour la mise à disposition d'un disjoncteur à rails.

\section{Références}

[1] Fiévet C., Barrault M., Petit P., Chevrier P., Fleurier C. and André V., J. Phys. D: APPL. Phys. 30 (1997) 2991-2999. 